\section{(2) OPEN ACCESS}

\title{
Effect of a formulated eye drop with Leptospermum spp honey on tear film properties
}

\author{
Jacqueline Tan (ㅁ), Tianni Jia, Roslyn Liao, Fiona Stapleton
}

School of Optometry and Vision Science, University of New South Wales, Sydney, New South Wales, Australia

\section{Correspondence to} Dr Jacqueline Tan, University of New South Wales School of Optometry and Vision Science, Sydney, NSW2052, Australia; jacqueline.tan@unsw.edu.au

Received 27 August 2019 Revised 10 December 2019 Accepted 27 December 2019
Check for updates

(C) Author(s) (or their employer(s)) 2020. Re-use permitted under CC BY-NC. No commercial re-use. See rights and permissions. Published by BMJ.

To cite: Tan J, Jia T, Liao R, et al. Br J Ophthalmol 2020;104:1373-1377.

\section{ABSTRACT}

Aim To evaluate the effects of a proprietary formulated eye drop with Leptospermum spp honey versus a conventional lubricant eye drop on tear film properties in subjects with symptoms related to dry eye disease after 28 days of treatment.

Methods Forty-six subjects with symptoms related to dry eye (Ocular Surface Disease Index (OSDI) score $>12$ ) were enrolled and randomly assigned to receive either the test formulated eye drop (Optimel by Melcare Biomedical Pty Ltd) or control eye drops (Alcon, USA) in this doublemasked study. Inferior lipid layer thickness (LLT), tear film evaporation rate (TER), fluorescein tear film break-up time (TBUT), corneal staining and subjective symptoms (OSDI and visual analogue scales (VAS)) were measured before and after 28 days of instilling the eye drops.

Results Forty-two subjects completed the study (21 subjects in each group). After 28 days of treatment, TER showed a significantly greater reduction with the formulated eye drop compared with the control $(p=0.01)$. TBUT showed a slight but not statistically significant increase with the formulated eye drop compared with the control $(p=0.06)$, and a significantly greater reduction (improvement) in OSDI scores was observed with the formulated eye drop compared with the control $(p=0.01)$. No significant differences were found between the two groups for inferior LLT, corneal staining and any of the VAS scores.

Conclusions The formulated eye drops were effective in reducing tear film evaporation rate and were more effective for improving symptoms of dry eye compared with the control eye drops after 28 days of treatment. Trial registration number NCT03622619.

\section{INTRODUCTION}

The involvement of inflammation as a core mechanism in dry eye disease is widely recognised. ${ }^{1}{ }^{2}$ Patients with dry eye display a higher concentration of proinflammatory markers in the tear fluid including cytokines and chemokines. ${ }^{3-5}$ However, treatment options for dry eye disease remain largely limited to tear replacement with ocular lubricants ${ }^{6}$ - products that do not target the underlying pathophysiology.

Leptospermum spp (Manuka) honey is a natural therapy that has antimicrobial, ${ }^{7}$ antioxidant and anti-inflammatory properties. ${ }^{8} 9$ In chronic ocular conditions characterised by bacterial overcolonisation, where ocular inflammatory responses are implicated, ${ }^{10}$ decreasing the bacterial load using topical antibiotics is effective for providing symptomatic relief. ${ }^{11}$ Given concerns regarding the development of drug resistance with antibiotics, and the risk of side effects with corticosteroid use, Manuka honey shows promise as an alternative or adjunct management strategy in the treatment of inflammation in dry eye disease. ${ }^{12}$

Optimel Manuka+ Dry Eye Drops is a proprietary formulated eye drop with $16 \%$ Leptospermum spp honey and is at present, along with the gel form ( $98 \%$ Leptospermum spp honey), the only formulated eye drop with honey commercially available in either Australia or the UK. ${ }^{13}$ Formulated eye drops with honey have been shown to improve ocular comfort when used twice daily in as little as 2 weeks in contact lens wearers ${ }^{14}$ and after 8 weeks in subjects with meibomian gland dysfunction. ${ }^{13}$ No significant differences were observed in objective measures of dry eye after 2 weeks of use, which may have been related to the short duration of treatment. ${ }^{14}$ However, measurable improvements in tear film break-up time, tear osmolarity and meibomian expressibility were observed after 8 weeks of treatment. ${ }^{13}$

Therefore, the purpose of this study was to evaluate the effects of a formulated eye drop with Leptospermum spp honey versus a conventional ocular lubricant on tear film properties, ocular surface staining and comfort in subjects with symptoms related to dry eye disease after 28 days of treatment, to determine whether measurable changes to objective tear film characteristics in particular can be detected earlier than after 8 weeks of treatment.

\section{MATERIALS AND METHODS \\ Study design}

This was a prospective, randomised, double-masked study comparing various tear film properties and subjective comfort after 28 days of treatment three times daily with the test formulated eye drop with Leptospermum spp honey $(165 \mathrm{mg} / \mathrm{g}$; Optimel Manuka+ Dry Eye Drops developed by Melcare Biomedical Pty Ltd) and control conventional ocular lubricant (polyethylene glycol $4000.4 \%$, propylene glycol 0.3\%; Alcon Laboratories, USA).

Participants were recruited from the local population at the investigational site (School of Optometry and Vision Science, University of New South Wales (UNSW)) by posting the approved study advertisement on UNSW noticeboards and websites. All procedures were conducted in accordance with the Declaration of Helsinki.

\section{Study participants}

A minimum sample size of 38 participants (19 in each group) was required to detect a change in tear 
film lipid layer thickness of $27 \mathrm{~nm}$, with a SD of $29 \mathrm{~nm}^{15}$ as measured using the LipiView II Ocular Surface Interferometer with $95 \%$ confidence and $80 \%$ power. Measurable changes to lipid layer thickness were anticipated given that Albietz and Schmid demonstrated improvements in meibomian gland expressibility and meibum quality after 8 weeks of treatment with the same formulated eye drop with honey as evaluated in this study. ${ }^{13}$ Subjects were eligible to participate if they met all of the inclusion criteria and none of the exclusion criteria. Inclusion criteria included minimum 18 years of age; a minimum dry eye classification of 'mild' according to the Ocular Surface Disease Index (OSDI) questionnaire ${ }^{16}$; and no planned changes to diet, including food, tea and supplements known to have anti-inflammatory properties. Exclusion criteria included allergy to honey products; soft contact lens wear during the study and within 1 week prior to enrolment; gas-permeable contact lens wear including orthokeratology during the study and within 2 weeks prior to enrolment; active eye infection, inflammation or allergy; eye injury or surgery within the past 6 months; any systemic disease that may affect ocular physiology; use of any corticosteroids, immunosuppressant or antihistamine medications 12 weeks prior to enrolment and during the study; pregnancy or lactating; and sensitivity to flashing strobe-like lights.

\section{Study procedures}

Study participants attended two study visits: baseline and following 28 days of treatment. At the baseline visit, study participants completed the OSDI questionnaire, visual acuity was measured for safety purposes using standard computerised letter charts, ${ }^{17}$ and slit-lamp biomicroscopy (Zeiss SL-120; Carl Zeiss Meditech, Jena, Germany) was performed to determine participant eligibility. At baseline, the following tear film measurements were obtained in order from the least to most invasive procedure: (1) tear film inferior lipid layer thickness (LLT; nm) with a LipiView II (Johnson and Johnson Vision, USA); (2) tear evaporation rate (TER; grams/square metre/ hour $\left(\mathrm{gm}^{-2} \mathrm{~h}\right)$ ) using a modified VapoMeter ${ }^{18}$ (http://www.delfin tech.com/en/vapometer/) including the gentle application of petroleum jelly (Vaseline, http://www.unilever.com.au/brands-in-action /detail/Vaseline/299339/) to the upper eyelids and surrounding area to minimise the effect of skin evaporation; (3) fluorescein tear break-up time (TBUT; s) (Opti-Strip-FL; Optimed, Lane Cove West, NSW, Australia) viewed with a yellow Wratten filter (No. 12; Kodak) and cobalt light of the slit-lamp biomicroscope. The extent of fluorescein corneal staining was graded for each quadrant using the CCLRU (or IER or BHVI) grading scale, ${ }^{19}$ and the results for each quadrant were averaged to obtain an overall score for each eye. Three consecutive TBUT measurements for each eye were taken and averaged. Objective measurements were conducted by a single masked investigator, while an unmasked investigator randomised participants to receive either the test or control eye drops, and conducted the eye drop dispensation and collection on completion of the study. Attempts were made to mask the study participants by over-labelling the eye drop bottles with generic information including the eye drop usage, expiry and study code. Ambient room temperature and humidity were kept to a constant $22^{\circ} \mathrm{C}$ and within a range of $35 \%$ to $50 \%$, respectively, as these environmental factors have been shown to affect tear film characteristics. ${ }^{20} 21$

On completion of the baseline visit, participants were dispensed with the study eye drops to be used three times a day. Participants were required to refrain from using eye drops for at least 4 hours prior to attending for clinical examination. The follow-up visit was scheduled 28 days ( \pm 4 days) later at approximately the same time of day, and the same measurements as conducted at baseline were repeated. The study eye drops were collected, and subjects were exited from the study.

To gauge compliance to treatment, the weight of a single unused bottle of each type of eye drop was measured and recorded. One hundred sixty-eight drops of each type of eye drop were weighed to determine the expected weight of returned eye drops if the participant was $100 \%$ compliant to treatment ( 28 days $\times 3$ times daily usage $\times 2$ eyes). Each bottle collected at the follow-up visit was reweighed, and based on the remaining weight, estimation of the number of drops used throughout the study period was estimated. Compliance was rated as follows: 'excellent' if instilled $>2.5$ times per day; 'good' if 2 to 2.5 ; 'fair' if 1.5 to 2 ; 'poor' if $<1.5$.

\section{Statistical analysis}

Data from the right and left eyes were not statistically different $(p>0.05)$ and were therefore averaged for the analysis. Normality was assessed using the Shapiro-Wilk test. Independent t-tests were used for comparison of continuous normally distributed data between the test and control groups. Non-parametric data were analysed using Mann-Whitney U test. Proportions were compared using $\chi^{2}$ tests and associations were examined using Pearson's correlation. The level of significance was set at alpha $=0.05$. Statistical analysis was performed using SPSS software V.22.0. The difference between the 28 days and baseline data was used to quantify the treatment effect.

\section{RESULTS}

A total of 46 participants were enrolled to ensure a minimum of 38 participants completed the study. Three participants were lost to follow-up (one in the formulated eye drop group and two in the control group) and one participant in the formulated eye drop group withdrew from the study due to stinging associated with the eye drop. Therefore, 42 participants successfully completed the study. The demographics and baseline characteristics of participants who completed the study are shown in table 1 . There were no significant differences in the baseline characteristics between the two groups, although participants assigned to the control group tended to report more blurry vision at baseline compared with participants assigned to the formulated eye drop group (38.9 vs $22.9, \mathrm{p}=0.08)$.

\begin{tabular}{llll}
\hline Table 1 & Baseline participant characteristics & \\
& $\begin{array}{l}\text { Formulated eye drop } \\
(\mathbf{n}=\mathbf{2 1})\end{array}$ & $\begin{array}{l}\text { Control eye drop } \\
(\mathbf{n}=\mathbf{2 1})\end{array}$ & P value \\
\hline Age (years) & $22.2 \pm 3.5$ & $20.6 \pm 3.2$ & 0.12 \\
\hline Gender (M:F) & $9: 12$ & $5: 16$ & - \\
\hline Inferior LLT (nm) & $68.9 \pm 23.2$ & $68.5 \pm 19.2$ & 0.96 \\
\hline TER (gm ${ }^{-2}$ h) & $41.7 \pm 13.7$ & $35.9 \pm 12.5$ & 0.18 \\
TBUT (s) & $5.1 \pm 1.8$ & $6.0 \pm 2.9$ & 0.13 \\
Corneal staining (0-4) & $0.5 \pm 0.5$ & $0.3 \pm 0.4$ & 0.16 \\
OSDI (0-100) & $33.7 \pm 12.4$ & $28.2 \pm 12.3$ & 0.15 \\
Symptoms (0-100)* & & & \\
\hline Burning/stinging & $23.3 \pm 21.6$ & $22.9 \pm 23.4$ & 0.74 \\
\hline $\begin{array}{l}\text { Grittiness/foreign body } \\
\text { sensation }\end{array}$ & $37.1 \pm 26.6$ & $29.0 \pm 27.0$ & 0.26 \\
\hline Dryness & $59.2 \pm 24.5$ & $55.9 \pm 24.6$ & 0.57 \\
\hline Blurry vision & $22.9 \pm 23.9$ & $38.9 \pm 31.3$ & 0.08 \\
\hline Overall discomfort & $27.3 \pm 22.3$ & $24.6 \pm 26.7$ & 0.52 \\
\hline
\end{tabular}

* $0=$ none, $100=$ maximum.

LLT, lipid layer thickness; OSDI, Ocular Surface Disease Index; TBUT, tear break-up time; TER, tear evaporation rate. 
Table 2 Mean and SD of change in ocular comfort and tear film characteristics after 28 days of treatment ( 28 days minus baseline)

\begin{tabular}{lccl}
\hline & $\begin{array}{l}\text { Formulated eye drop } \\
(\mathbf{n}=\mathbf{2 1})\end{array}$ & $\begin{array}{l}\text { Control eye drop } \\
(\mathbf{n}=\mathbf{2 1})\end{array}$ & P value \\
\hline Inferior LLT (nm) & $-5.1 \pm 22.2$ & $-3.2 \pm 21.8$ & 0.65 \\
\hline TER (gm $\left.{ }^{-2} \mathrm{~h}\right)$ & $-10.8 \pm 16.7$ & $0.7 \pm 10.8$ & 0.014 \\
\hline TBUT (s) & $2.0 \pm 3.0$ & $0.0 \pm 3.1$ & 0.055 \\
\hline Corneal staining (0-4) & $-0.2 \pm 0.6$ & $0.0 \pm 0.5$ & 0.64 \\
\hline OSDI (0-100) & $-19.6 \pm 10.9$ & $-10.7 \pm 7.1$ & 0.005 \\
\hline Symptoms (0-100) & & & \\
\hline Burning/stinging & & & \\
\hline Grittiness/foreign & $-10.7 \pm 23.2$ & $-8.9 \pm 18.6$ & 0.90 \\
\hline Body sensation & $-20.8 \pm 20.4$ & $-15.0 \pm 17.9$ & 0.30 \\
\hline Dryness & $-35.4 \pm 22.1$ & $-32.2 \pm 19.2$ & 0.66 \\
\hline Blurry vision & $-13.6 \pm 22.5$ & $-20.6 \pm 29.8$ & 0.14 \\
\hline Overall discomfort & $-14.7 \pm 15.1$ & $-10.7 \pm 17.5$ & 0.30 \\
\hline
\end{tabular}

LLT, lipid layer thickness; OSDI, Ocular Surface Disease Index; TBUT, tear break-up time; TER, tear evaporation rate.

Overall, both eye drops were well tolerated. No adverse events were reported in the control group, but one case of ocular stinging was reported in the formulated eye drop group, which led to the participant withdrawing from the study.

Table 2 summarises the change in inferior LLT, TER, TBUT, corneal staining, OSDI score and visual analogue scale (VAS) symptoms from baseline after 28 days of daily use with the formulated eye drop with and the control eye drops.

After 28 days of treatment, a significant reduction in TER was observed with the formulated eye drop compared with the control eye drop $\left(-10.8 \pm 16.8\right.$ vs $\left.0.7 \pm 10.8 \mathrm{gm}^{-2} \mathrm{~h}, \mathrm{p}=0.01\right)$, while a corresponding increase in TBUT with the formulated eye drop was observed, although this did not reach statistical significance (2.0 \pm 3.0 vs 0.0 vs $3.1 \mathrm{~s}, \mathrm{p}=0.06$ ). However, no significant difference was observed in inferior LLT between the baseline and 28-day visits for either the formulated eye drop $(p=0.29)$ and the control eye drops $(p=0.61)$, and subsequently no significant difference was observed for change in inferior LLT between the two groups after 28 days of treatment $(-5.1 \pm 22.2$ vs $-3.2 \pm 21.8, \mathrm{p}=0.65)$.

Furthermore, after 28 days of treatment, both eye drops were effective in reducing (improving) ocular symptoms measured using the OSDI and VAS questionnaires $(\mathrm{p} \leq 0.01)$. However, a significantly greater reduction in OSDI scores was observed with the formulated eye drop compared with the control eye drop $(-19.6 \pm 10.9$ vs $-10.7 \pm 7.1, p=0.01)$. There were no significant differences in symptom improvement between the two eye drops measured using the VAS questionnaires $(p>0.05)$.

Compliance to each study eye drop was estimated based on the weight of bottles returned by 38 participants $(19$ formulated and 19 control). The remaining bottles ( 2 of each type) not returned were reported to be lost. There were no significant differences in compliance to treatment between the two groups $(\mathrm{p}=0.17$; figure 1$)$.

Furthermore, no significant associations were found between participant compliance and change in OSDI score, TER or TBUT, or between change in TER and change in TBUT after 28 days of study eye drop use $(\mathrm{p}>0.05)$.

\section{DISCUSSION}

The formulated eye drop with Leptospermum spp honey was effective in reducing tear film evaporation rate after 28 days of daily use and was more effective for improving symptoms related

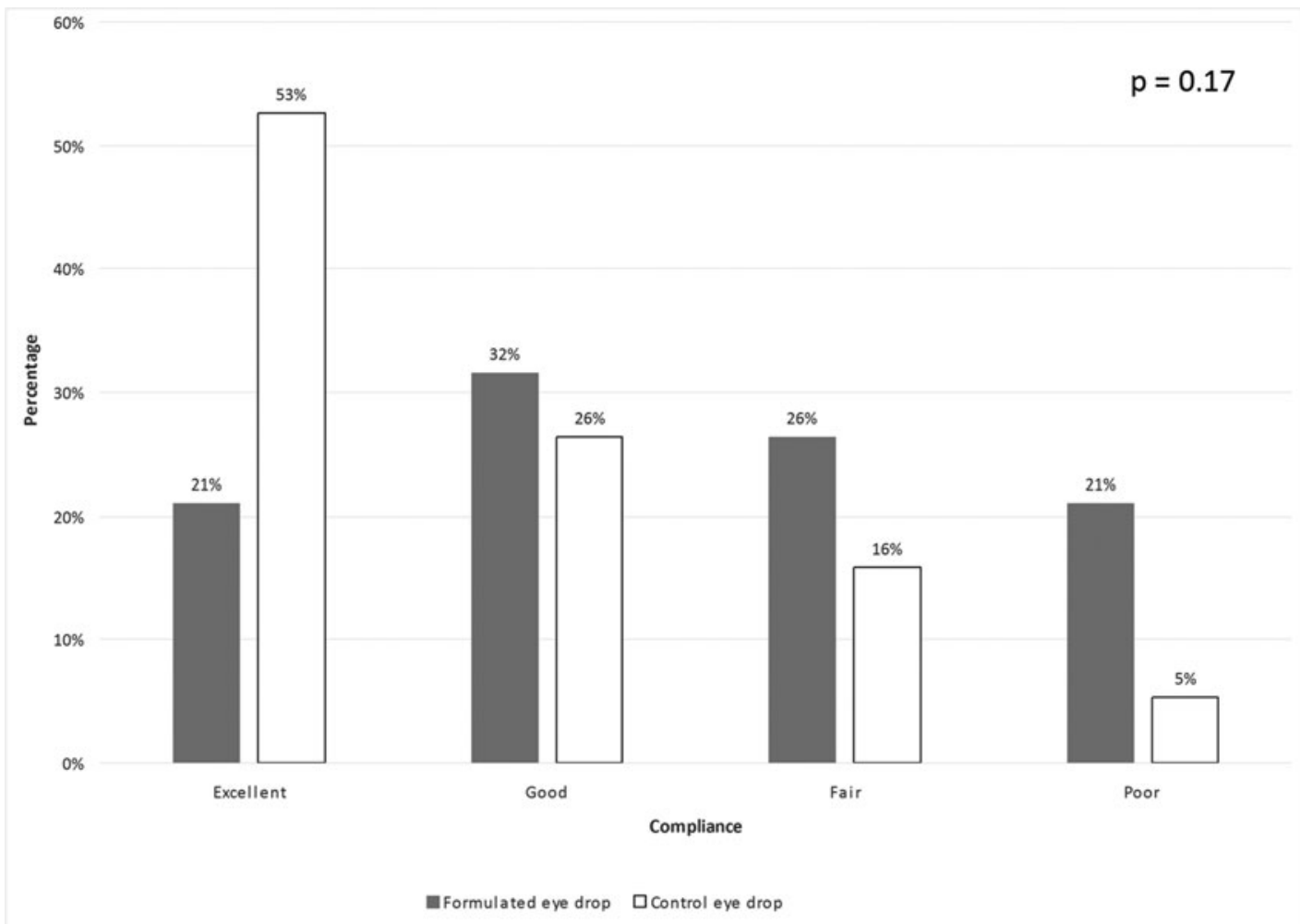

Figure 1 Proportion of subjects compliant to each eye drop. 
to dry eye disease compared with the control conventional eye drops evaluated in this study.

Higher TER have been reported in patients with dry eye, ${ }^{22} 23$ and given tear film thinning and break-up occur mainly as a result of evaporation from the tear film, ${ }^{24}$ slowing TER is important to improve tear film stability. In this study, the formulated eye drop with honey was effective for reducing TER.

Fluorescein TBUT showed a slight but not statistically significant increase after 28 days of daily use of the formulated eye drop only. Post hoc calculation showed $56.5 \%$ power to detect a difference in this study sample (https:/clincalc.com/stats/ Power.aspx). A sample size of 38 participants in each group would have been required to reach $80 \%$ power (http://statula tor.com/SampleSize/ss2M.html), which is consistent with subject numbers in a previous study that demonstrated a significant improvement in fluorescein TBUT after 8 weeks of use of the same formulated eye drop with honey as evaluated in this study. ${ }^{13}$ Given that the application of fluorescein destabilises the tear film, ${ }^{25}$ it may have been preferable to use non-invasive techniques to measure TBUT. However, the potential effect of fluorescein volume on TBUT was minimised in this study, by shaking excess fluid off the strips prior to application, which produces measurements more comparable with non-invasive methods. ${ }^{25}$

Albeitz and Schmid also reported improvements in corneal staining, osmolarity and meibum quality with the use of formulated eye drops with honey after 8 weeks of use. ${ }^{13}$ Wong et al reported a significant reduction in tear meniscus height and Schirmer I score following use of the same formulated eye drops as evaluated in this study, when used twice a day for 2 weeks in contact lens wearers. ${ }^{14}$ However, when the data were considered only for those participants classified as having symptoms of at least mild dry eye according to OSDI criteria, ${ }^{16}$ these changes were no longer apparent.

The findings for the control group in this study where TER remained unchanged after 4 weeks of treatment are consistent with previous evaluations of conventional tear film supplements, where no significant change in TER was observed after 4 weeks of treatment with either of the two formulations containing emulsified lipids, or the supplement containing a standard aqueous polymer, when TER was measured using the ServoMed EP-3 Evaporimeter. $^{26}$

Formulated eye drops with honey have been demonstrated to improve ocular comfort in as little as 2 weeks ${ }^{14}$ and after 8 weeks of treatment. ${ }^{13}$ In this study, both of the study eye drops were effective for improving comfort after 28 days of treatment, but a greater improvement in comfort was observed with the formulated eye drop. This result is promising, given previous reports of 'stinging' with topical application of antibacterial honey. ${ }^{7}$ Consideration should be given to the utility of counselling patients prior to prescribing formulated eye drops with honey, regarding the potential for initial discomfort associated with use, as a way to mitigate poor compliance.

The formulated eye drops with Leptospermum spp honey are effective for significantly reducing total lid margin bacterial colony counts after 8 weeks of use in subjects with meibomian gland dysfunction, where no change in total colony count was observed with the control conventional therapy, which was the same ocular lubricant evaluated in this study. ${ }^{13}$ Furthermore, a significant reduction in the inflammatory marker tear MMP-9 was observed in subjects treated with the two types of formulated eye drops with honey only. ${ }^{13}$ In blepharitis, the cause of inflammation appears to be bacterial by-products rather than the bacteria themselves. ${ }^{27}$ These findings suggest that the formulated eye drops with honey may be effective for the treatment of dry eye disease as a result of the dual antibacterial and anti-inflammatory properties previously reported. However, this proposed mechanism of action warrants further exploration.

No significant changes in inferior LLTwere observed after 28 days of daily use with either of the study eye drops. This result is in contrast to a previous study, which evaluated the same formulated eye drops and the conventional control therapy as evaluated in this study, and showed improvements in meibum quality and gland expressibility after 8 weeks of use. ${ }^{13}$ This may be attributed to the lack of correlation between LLT and meibum quality scores, which was recently reported by Sang et al. ${ }^{28}$ While areas of tear break-up are often associated with thin lipid, it has been shown that this is not always the case ${ }^{29}$ which supports that the tear lipid is a poor barrier to evaporation if the composition and/or structure are defective. The lack of change in inferior LLT in this study, despite improvements to TER and TBUT with the formulated eye drops, seems to suggest that inferior LLT is not the primary driver in minimising TER. However, the LipiView II interferometer measures inferior LLT only, which may not be representative of LLT across the entire ocular surface. Furthermore, the study sample size was calculated based on inferior LLT, but no change in inferior LLTwas observed. Sample size calculation was powered for a specified change in LLT using a different device to that used in this study (Stroboscopic Video Colour Microscope (SVCM) vs LipiView II). Although both instruments use interferometric methods to measure LLT, the areas of the tear film evaluated differ between these two instruments: the SVCM images a circular area of $6 \mathrm{~mm}$ diameter, ${ }^{15}$ whereas the LipiView images the inferior region of the tear film. ${ }^{30}$ Therefore, this study may not have been appropriately powered to detect change in LLT. It would have been more appropriate to power the study using a more relevant clinical parameter.

In this study, no significant change in corneal staining was observed with either eye drop after 4 weeks of treatment, whereas Albietz and Schmid ${ }^{13}$ showed a significant reduction in interpalpebral corneal and conjunctival staining after 8 weeks of daily use with the formulated eye drops with Leptospermum spp honey (16\%). This difference may be attributable to the different grading scale used by Albietz et al (Oxford Scheme (0 to 15)), ${ }^{31}$ which included conjunctival staining, the low levels of corneal staining observed in this study at baseline, or small differences in the severity of dry eye between the subject populations, as the participants in Albeitz's study assigned to the formulated eye drop with honey had, on average, a numerically higher OSDI score compared with the participants assigned to the formulated eye drop in this study at baseline (38.2 \pm 15.6 vs $33.7 \pm 12.4$, respectively).

Self-reported compliance to the same formulated eye drops with honey as evaluated in this study, when prescribed to contact lensrelated dry eye subjects, found $40 \%$ were rated as 'excellent' (two drops per day nearly every day as recommended), 35\% 'good' (one or two drops per day most days), 20\% 'fair' (one drop per day most days) and 5\% 'poor' (one or two drops when needed only). ${ }^{14}$ Fiftyeight per cent of participants in this study were rated as having 'good' to 'fair' compliance (1.5 to 2.5 drops per day) to treatment with the formulated eye drops based on the weight of returned bottles, and therefore actual eye drop usage was comparable with the study by Wong et al. However, the positive results in this study suggest that two times daily dosing is adequate to achieve improvements in subjective comfort and tear film parameters.

Shortcomings of the study included incomplete double masking and the choice of control eye drop. Difficulty in maintaining participant masking due to the distinctive smell and taste of the formulated eye drops with honey has previously been reported. ${ }^{14}$ While it is possible that this may have influenced the subjective comfort scores, the objective tear film measurements were 
conducted by a masked investigator to eliminate any potential bias. Furthermore, the use of the conventional lubricant eye drop as the comparator was not ideal. It would have been preferable to compare the formulated eye drops with honey against the vehicle only, to determine the true effect of honey on tear film properties and symptoms. It is possible that removal of petroleum jelly from the eyelids following TER measurements may have affected subsequent TBUT measurements. However, to minimise this effect, petroleum jelly was gently removed by wiping the superficial skin surface with minimal pressure. In addition, while it is possible that migration of petroleum jelly into the tear film may have affected tear stability measures, this did not appear to occur given that none of the study participants reported any comfort or vision-related issues following application or removal. The inclusion of ocular swabs and assessment of other tear film properties such as inflammatory markers and tear osmolarity might also have been useful for confirming potential mechanisms of action. It is also unclear whether these findings can be generalised to the wider population, given the overall young average age of participants in this study. Future larger-scale studies, to include a wider age range of participants, would be worthwhile for elucidating whether the formulated eye drops with honey are effective in a broader population and to determine the effect on different severities of dry eye.

The formulated eye drops with Leptospermum spp honey were effective for reducing tear film evaporation rate and reducing ocular symptoms related to dry eye compared with the control conventional eye drops evaluated in this study. Formulated eye drops with honey should be considered in the management of dry eye.

Acknowledgements The authors wish to thank Designs for Vision, Australia, a subsidiary of Melcare Biomedical Pty Ltd for providing the formulated eye drops with Leptospermum spp honey used in this study. We also wish to thank Dr Jaya Sowjanya Siddireddy for providing statistical support.

Contributors All authors took an active part in the design, conduct, data analysis, and publication drafting and approval.

Funding The authors have not declared a specific grant for this research from any funding agency in the public, commercial or not-for-profit sectors.

Competing interests None declared.

Patient consent for publication Obtained.

Ethics approval University of New South Wales (UNSW) Human Research Ethics Committee (HC180256).

Provenance and peer review Not commissioned; externally peer reviewed.

Data availability statement Data are available on request. All data relating to the study are published. Any requests for data can be made to the corresponding author and are subject to ethics approval.

Open access This is an open access article distributed in accordance with the Creative Commons Attribution Non Commercial (CC BY-NC 4.0) license, which permits others to distribute, remix, adapt, build upon this work non-commercially, and license their derivative works on different terms, provided the original work is properly cited, appropriate credit is given, any changes made indicated, and the use is noncommercial. See: http://creativecommons.org/licenses/by-nc/4.0/.

\section{ORCID iD}

Jacqueline Tan http://orcid.org/0000-0003-4986-4945

\section{REFERENCES}

1 Craig JP, Nichols KK, Akpek EK, et al. TFOS DEWS II definition and classification report. Ocul Surf 2017;15:276-83.
2 Wei Y, Asbell PA. The core mechanism of dry eye disease is inflammation. Eye Contact Lens 2014:40:248-56.

3 Solomon A, Dursun D, Liu Z, et al. Pro- and anti-inflammatory forms of interleukin-1 in the tear fluid and conjunctiva of patients with dry-eye disease. Invest Ophthalmol Vis Sci 2001;42:2283-92.

4 Massingale ML, Li X, Vallabhajosyula M, et al. Analysis of inflammatory cytokines in the tears of dry eye patients. Cornea 2009;28:1023-7.

5 Tishler M, Yaron I, Geyer 0, et al. Elevated tear interleukin-6 levels in patients with Sjögren syndrome. Ophthalmology 1998;105:2327-9.

6 Jones L, Downie LE, Korb D, et al. TFOS DEWS II management and therapy report. Ocul Surf 2017:15:575-628.

7 Albietz JM, Lenton LM. Effect of antibacterial honey on the ocular flora in tear deficiency and meibomian gland disease. Cornea 2006;25:1012-9.

8 Tonks AJ, Cooper RA, Jones KP, et al. Honey stimulates inflammatory cytokine production from monocytes. Cytokine 2003;21:242-7.

9 Almasaudi SB, El-Shitany NA, Abbas AT, et al. Antioxidant, anti-inflammatory, and antiulcer potential of manuka honey against gastric ulcer in rats. Oxid Med Cell Longev 2016;2016:3643824

10 Craig JP, Rupenthal ID, Seyfoddin A, et al. Preclinical development of MGO Manuka Honey microemulsion for blepharitis management. BMJ Open Ophthalmol 2017;1:e000065.

11 Lindsley K, Matsumura S, Hatef E, et al. Interventions for chronic blepharitis. Cochrane Database Syst Rev 2012:CD005556.

12 Craig JP, Wang MTM, Ganesalingam K, et al. Randomised masked trial of the clinical safety and tolerability of $\mathrm{MgO}$ manuka honey eye cream for the management of blepharitis. BMJ Open Ophthalmol 2017;1:e000066.

13 Albietz JM, Schmid KL. Randomised controlled trial of topical antibacterial Manuka (Leptospermum species) honey for evaporative dry eye due to meibomian gland dysfunction. Clin Exp Optom 2017:100:603-15.

14 Wong D, Albietz JM, Tran H, et al. Treatment of contact lens related dry eye with antibacterial honey. Cont Lens Anterior Eye 2017:40:389-93.

15 Fogt JS, Kowalski MJ, King-Smith PE, et al. Tear lipid layer thickness with eye drops in meibomian gland dysfunction. Clin Ophthalmol 2016;10:2237-43.

16 Miller KL, Walt JG, Mink DR, et al. Minimal clinically important difference for the ocular surface disease index. Arch Ophthalmol 2010;128:94-101.

17 Ehrmann K, Fedtke C, Radić A. Assessment of computer generated vision charts. Contact Lens and Anterior Eye 2009;32:133-40.

18 Rohit A, Ehrmann K, Naduvilath T, et al. Validating a new device for measuring tear evaporation rates. Ophthalmic Physiol Opt 2014;34:53-62.

19 Terry RL, Schnider CM, Holden BA, et al. CCLRU standards for success of daily and extended wear contact lenses. Optom Vis Sci 1993;70:234-43.

20 Madden LC, Tomlinson A, Simmons PA. Effect of humidity variations in a controlled environment chamber on tear evaporation after dry eye therapy. Eye Contact Lens 2013:39:169-74

21 Abusharha AA, Pearce El, Fagehi R. Effect of ambient temperature on the human tear film. Eye Contact Lens 2016;42:308-12.

22 Mathers WD, Daley TE. Tear flow and evaporation in patients with and without dry eye. Ophthalmology 1996;103:664-9.

23 Rolando M, Refojo MF, Kenyon KR. Increased tear evaporation in eyes with keratoconjunctivitis sicca. Arch Ophthalmol 1983;101:557-8.

24 Willcox MDP, Argüeso P, Georgiev GA, et al. TFOS DEWS II tear film report. Ocul Surf 2017;15:366-403.

25 Mooi JK, Wang MTM, Lim J, et al. Minimising instilled volume reduces the impact of fluorescein on clinical measurements of tear film stability. Cont Lens Anterior Eye 2017:40:170-4.

26 Martin E, Oliver KM, Pearce El, et al. Effect of tear supplements on signs, symptoms and inflammatory markers in dry eye. Cytokine 2018;105:37-44.

27 O'Brien TP. The role of bacteria in blepharitis. Ocul Surf 2009; 7:S21-2.

28 Sang $X, L i$ Y, Yang L, et al. Lipid layer thickness and tear meniscus height measurements for the differential diagnosis of evaporative dry eye subtypes. Int I Ophthalmol 2018;11:1496-502.

29 King-Smith PE, Reuter KS, Braun RJ, et al. Tear film breakup and structure studied by simultaneous video recording of fluorescence and tear film lipid layer images. Invest Ophthalmol Vis Sci 2013:54:4900-9.

30 Eom Y, Lee J-S, Kang S-Y, et al. Correlation between quantitative measurements of tear film lipid layer thickness and meibomian gland loss in patients with obstructive meibomian gland dysfunction and normal controls. Am J Ophthalmol 2013;155:1104-10.

31 Bron AJ, Evans VE, Smith JA. Grading of corneal and conjunctival staining in the context of other dry eye tests. Cornea 2003;22:640-50. 